CLINICAL STUDY

\title{
Recent outcome of Graves' disease patients with papillary thyroid cancer
}

Yukiko Yano, Hiroshi Shibuya, Wataru Kitagawa, Mitsuji Nagahama, Kiminori Sugino, Kunihiko Ito and Koichi Ito Ito Hospital, 4-3-6 Jinngu-mae, Shibuya, Tokyo 150-8308, Japan

(Correspondence should be addressed to Y Yano; Email: geka@ito-hospital.jp)

\begin{abstract}
Objective: The objective was to evaluate the clinical behavior and outcome of 202 papillary thyroid cancers in Graves' disease patients during the period 1994-2004.

Design: This was a retrospective, non-randomized case-control study.

Methods: Since 1994, we have included an ultrasonogram of the neck in the initial examination of thyroid disease patients who consult our outpatient clinic. We evaluated the tumor status and longterm outcome of Graves' disease patients with thyroid cancer and of age- and tumor size-matched euthyroid papillary thyroid cancer patients as controls. Serum TSH receptor antibody (TRAb) was measured in the Graves' disease group.

Results: A total of 154 papillary thyroid cancers were diagnosed in the Graves' disease patients and were treated surgically. At surgery, no significant differences in multifocality, lymph node metastasis, or distant metastasis were found between the Graves' disease group and the euthyroid group. On the whole, the clinical course of the cancers in both the Graves' disease group and euthyroid group was relatively good. No significant correlations were found between the TRAb levels in the Graves' disease group and multifocality or the presence of lymph node metastasis. Papillary thyroid cancer was discovered as an incidental finding in $2 \%$ of the 2356 surgically treated Graves' disease patients, but none of them developed metastasis during the follow-up period.

Conclusion: The results in this series of patients do not support the claim that thyroid cancer is more aggressive in Graves' disease patients than in euthyroid patients.
\end{abstract}

European Journal of Endocrinology 157 325-329

\section{Introduction}

Whether thyroid cancer behaves more aggressively in patients with Graves' disease is a matter of controversy. A number of studies have suggested increased aggressiveness of papillary thyroid cancer and follicular thyroid cancer in Graves' disease patients (1-4), and many investigators have strongly suggested that the thyroid-stimulating antibodies cause greater aggressiveness of thyroid malignancy.

Thyroid-stimulating hormone (TSH) plays a role in thyroid growth and normal functioning and is involved in thyroid organogenesis, cell differentiation, iodide uptake, and triiodothyronine secretion (5). Thyroid nodules are common in patients with Graves' disease and raise concern about the possible presence of thyroid malignancy (6). Moreover, the thyroid-stimulating antibodies in Graves' disease stimulate the growth of thyroid cancer. Some investigators have reported a correlation between thyroid cancers that relapse after thyroidectomy and a high level of circulating thyroid-stimulating antibodies $(6,7)$.

Early detection of thyroid nodules has recently become possible by ultrasonography, and the increasing use of thyroid ultrasonography has revealed greater numbers of thyroid nodules that are not palpable. Small thyroid cancers are being discovered as incidental findings in Graves' disease patients $(4,8,9)$, and in some studies Graves' disease patients with a small thyroid cancer have been found to have a better outcome than patients with a small thyroid cancer manifested clinically $(9,10)$.

Since 1994, we have included an ultrasonogram in the initial examination of all thyroid disease patients who consult our hospital. The indications for surgery in Graves' disease patients include side effects of antithyroid medication, inability to take medication, an unusually large diffuse goiter, and malignancy or suspicious cytological findings in fine-needle biopsy specimens. We have been selecting surgical treatment for Graves' disease patients with thyroid cancer and avoiding prolonged medical treatment.

In this study, we evaluated the tumor status and longterm outcome of papillary thyroid cancer in Graves' disease patients regardless of the tumor size. This study was conducted at our hospital, which specializes in the treatment of thyroid disease, and about 1500 thyroid operations are performed annually. 


\section{Patients and methods}

\section{Graves' disease patients}

The diagnosis of Graves' disease was based on the classic criteria: hyperthyroidism with or without clinical ophthalmopathy, a diffuse homogeneous goiter on palpation, and the presence of TSH receptor antibody (TRAb) in the patient's serum. A scintiscan was performed on some patients as an additional examination to confirm the diagnosis of Graves' disease. During the period from 1994 to 2004, 32200 Graves' disease patients were seen in our outpatient clinic. Thyroid ultrasonography was performed in all patients with Graves' disease, and this was followed by fineneedle aspiration biopsy whenever thyroid cancer was suspected. Graves' disease patients with thyroid cancer were referred for surgery.

Surgery was performed in 2356 Graves' disease patients, and 277 of them had thyroid nodules. The cytological diagnosis was thyroid cancer in 166 patients and a suspicion of thyroid cancer in 12 patients. The histological diagnosis was papillary thyroid cancer in 157 patients, follicular thyroid cancer in 8 patients, and medullary thyroid cancer in 1 patient. Total thyroidectomy with lymph node dissection was performed in 4 of the patients with papillary thyroid cancer, subtotal thyroidectomy with lymph node dissection in 130 , lobectomy with lymph node dissection in 20, and lobectomy in 3. Histopathological examination of surgically resected thyroid glands resulted in the discovery of 48 papillary thyroid cancers. Three of the papillary thyroid cancer patients in this series were lost to follow-up.

Serum TRAb was measured by a binding inhibitory assay with a TRAb kit (RSR Limited, Cardiff, UK).

\section{Euthyroid control group}

The euthyroid control group consisted of 176 sex-, age-, and tumor size-matched papillary thyroid cancer patients who underwent surgery during the same period.

\section{Postoperative follow-up}

All patients were followed up postoperatively. Ultrasonography to detect residual disease in the thyroid gland and lymph node metastasis was routinely performed for 6-12 months after thyroidectomy. Regional lymph node metastasis was diagnosed by ultrasonography and cytodiagnosis. Patients with an elevated serum thyroglobulin level were examined for distant metastasis by diagnostic imaging; that is, radiography, computed tomography, or bone scan. The cause of death was determined by a review of the hospital records.
Table 1 The characteristics of the Graves' disease patients with papillary thyroid cancer and the euthyroid control group.

\begin{tabular}{lccc}
\hline & $\begin{array}{c}\text { Graves' } \\
\text { disease } \\
(n=154)\end{array}$ & $\begin{array}{c}\text { Euthyroid } \\
(n=176)\end{array}$ & $\boldsymbol{P}$ value \\
\hline Age (years) & & & \\
$\quad$ Range & $16-76$ & $17-75$ & 0.3106 \\
$\quad$ Median & 50.0 & 51.0 & \\
Gender (F/M) & $135 / 19$ & $151 / 25$ & 0.6187 \\
Diameter (cm) & & & \\
$\quad$ Range & $0.1-70.0$ & $0.2-75.0$ & 0.2647 \\
$\quad$ Median & 0.9 & 1.0 & \\
Multifocality & $54(34.4 \%)$ & $50(28.4 \%)$ & 0.1672 \\
LN metastasis & & & \\
Follow-up (month) & $69(44.8 \%)$ & $97(55.1 \%)$ & 0.1431 \\
$\quad$ Range & $10.6-120.0$ & $6.5-120.0$ & 0.4008 \\
$\quad$ Median & 50.4 & 53.6 & \\
\hline
\end{tabular}

aLymph node metastasis.

\section{Statistical analysis}

Statistical analysis was performed using Statistical Analysis System JMP software (SAS Institute, Cary, NC, USA). The log-rank test was used to evaluate differences among the groups. Analyses by the KaplanMeier method were performed with Statistical Analysis System JMP software.

\section{Results}

\section{Incidental papillary thyroid cancers}

Papillary thyroid cancer was diagnosed as an incidental finding in 48 Graves' disease patients who underwent

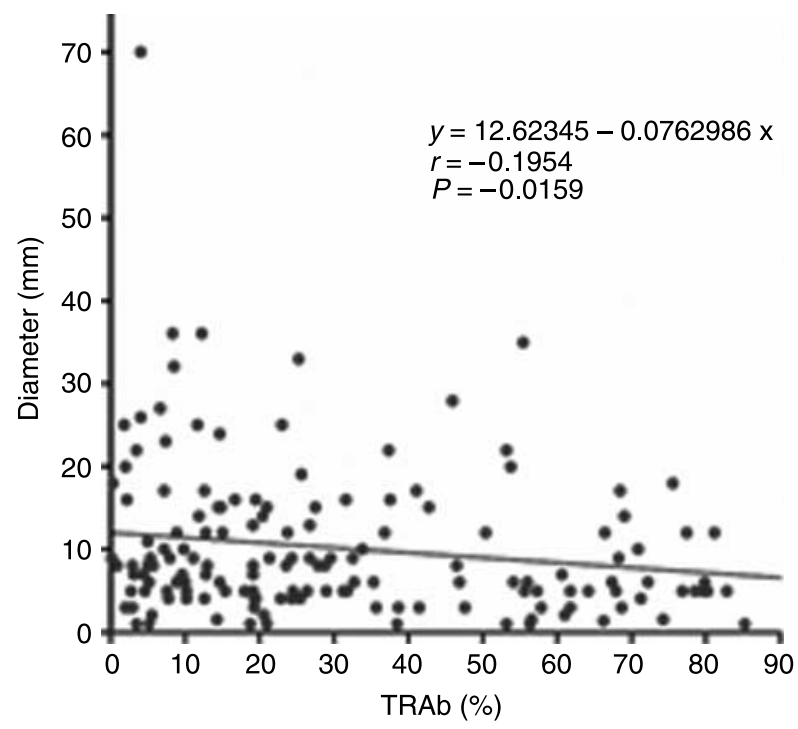

Figure 1 Correlation between thyroid receptor antibody (TRAb) values and tumor size in Graves' disease patients with papillary thyroid cancer. There was an inverse correlation between the TRAb values and tumor size $(r=-0.1954, P=0.0159)$. 


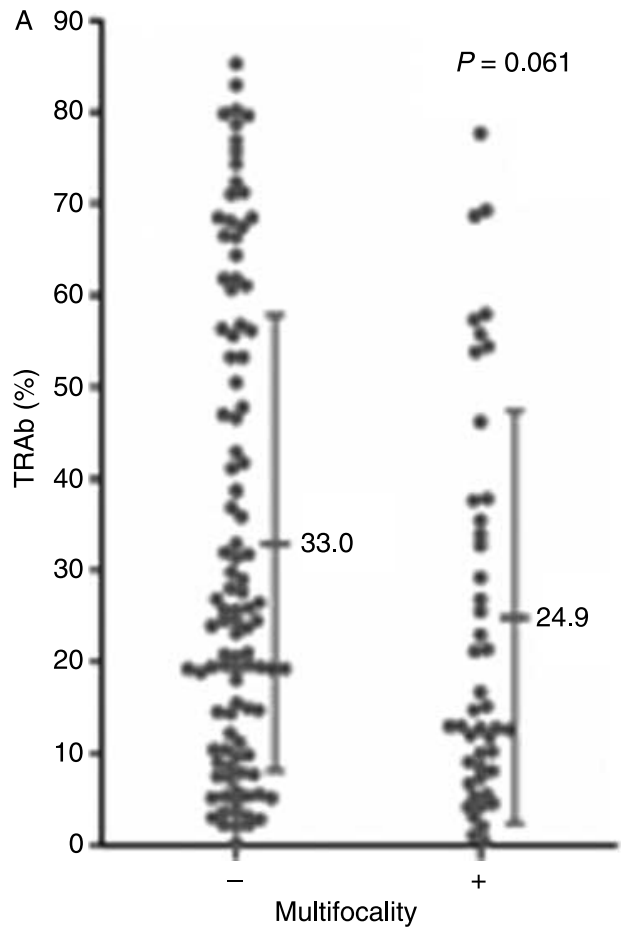

thyroidectomy. The frequency of incidental papillary thyroid cancer in the Graves' disease patients was $2.0 \%$ (48 patients with incidental thyroid cancer out of 2356 patients who underwent surgery for Graves' disease). None of the cancers were suspected preoperatively, and they were diagnosed during the pathological examination. The patients' median age at the time of surgery was 32.0 years (range 17-68). The median tumor diameter was $3.0 \mathrm{~mm}$ (range 1.0-15.0).

None of the patients were diagnosed with recurrence during the follow-up period. Four patients experienced a relapse of hyperthyroidism after surgery.

\section{Status of papillary thyroid cancers}

Papillary thyroid cancer was diagnosed preoperatively in 154 of the total Graves' disease patients. The characteristics and follow-up period of the Graves' disease patients with papillary thyroid cancer and the control group are summarized in Table 1. The median age was 50.0 years in the Graves' disease group and 51.0 years in the euthyroid group $(P=0.3106)$. Histological examination revealed multifocality in 53 (34.4\%) of the Graves' disease patients and lymph node metastasis in $69(44.8 \%)$ of the Graves' disease patients. At the time of surgery, there was no significant difference in multifocality or lymph node metastasis status between the Graves' disease and euthyroid groups $(P=0.1672,0.1431$ respectively). Two patients $(1.3 \%)$ in the Graves' disease group and three patients $(1.7 \%)$ in the control group had lung metastasis at the time of surgery $(P=0.7633)$.

Serum TRAb was measured in the Graves' disease group before medical treatment and was detected in every patient. There was an inverse correlation between the TRAb values and tumor size $(r=-0.195$, $P=0.0159$; Fig. 1). The mean TRAb value was $24.9 \%$ (normal range, $<10 \%$ ) in the patients with

Table 2 The occurrence of distant and lymph node metastasis of papillary thyroid cancer in the Graves' disease patients and euthyroid patients.

\begin{tabular}{|c|c|c|c|}
\hline & $\begin{array}{l}\text { Graves' } \\
\text { disease } \\
(n=154)\end{array}$ & $\begin{array}{l}\text { Euthyroid } \\
(n=176)\end{array}$ & $P$ value \\
\hline \multicolumn{4}{|c|}{ Patient with distant metastasis } \\
\hline At surgery & $2(1.3)^{\mathrm{a}}$ & $3(1.7)$ & 0.7633 \\
\hline $\begin{array}{l}\text { New cases during } \\
\text { the follow-up }\end{array}$ & $1(0.6)$ & $4(2.3)$ & 0.2284 \\
\hline Total cases & $3(1.9)$ & $7(4.0)$ & 0.2834 \\
\hline \multicolumn{4}{|c|}{ Patient with lymph node metastasis } \\
\hline At surgery & $69(44.8)$ & $97(55.1)$ & 0.1432 \\
\hline Relapses & $5^{b}(3.2)$ & $14^{\mathrm{b}}(8.0)$ & 0.1518 \\
\hline $\begin{array}{l}\text { New cases during } \\
\text { the follow-up }\end{array}$ & $1(0.6)$ & $4(2.3)$ & 0.1773 \\
\hline $\begin{array}{l}\text { Total relapses } \\
\text { and new cases }\end{array}$ & $6(3.9)$ & $18(10.2)$ & 0.0474 \\
\hline \multicolumn{4}{|c|}{ Patient follow-up status } \\
\hline $\begin{array}{l}\text { Persistent distant } \\
\text { metastasis }\end{array}$ & 2 & 6 & 0.2137 \\
\hline Death for cancer & 1 & 2 & 0.9245 \\
\hline
\end{tabular}

${ }^{a}$ Numbers in parentheses are percent.

'One Graves' disease patient and four euthyroid patients also had distant metastasis. 

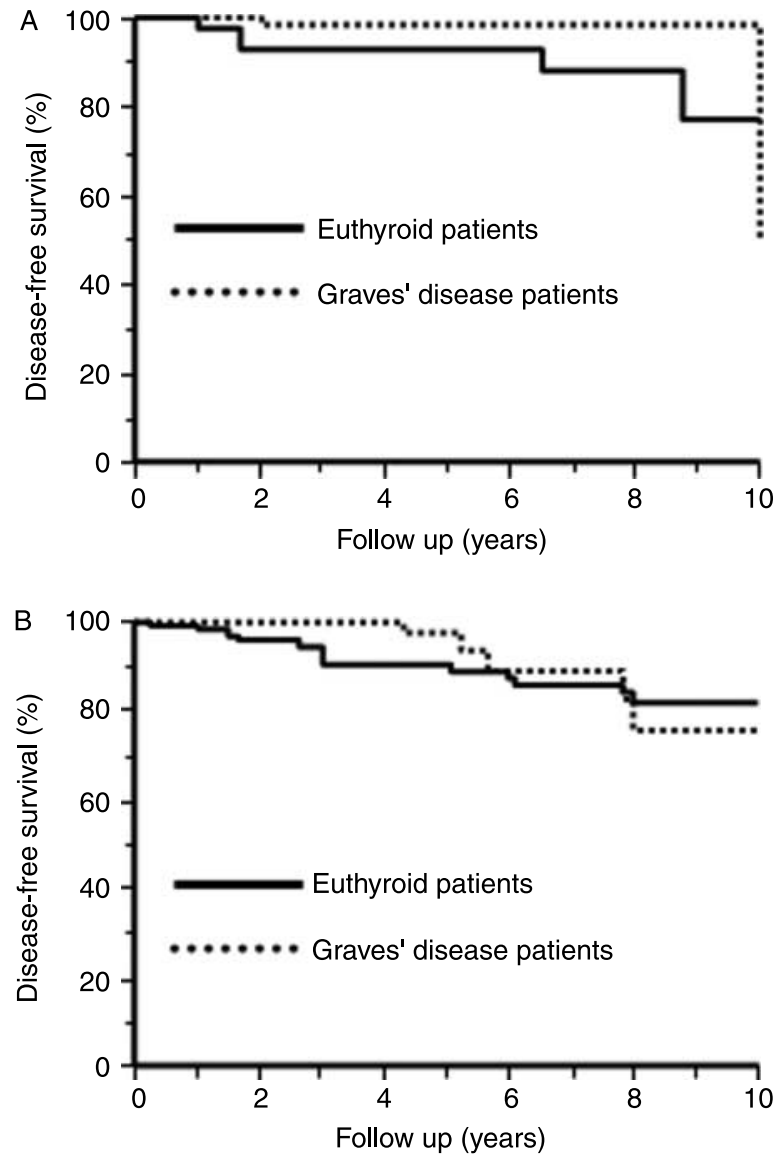

Figure 3 (A) Observed disease-free survival of patients with microcarcinoma in the Graves' disease group ( $n=90$, dotted line) and control group ( $n=89$, bold line) ( $P=0.1118$, log-rank test). (B) The disease-free survival rate of the patients with papillary thyroid cancer larger than $1 \mathrm{~cm}$ in the Graves' disease group ( $n=64$, dotted line) was not statistically different from that in the control group ( $n=87$, bold line; $P=0.7329$, log-rank test).

multifocality, and $33.0 \%$ in the patients without multifocality $(P=0.061$; Fig. 2A). The mean TRAb value was $25.4 \%$ in the patients with lymph node metastasis and $26.7 \%$ in the patients without lymph node metastasis $(P=0.779$; Fig. $2 \mathrm{~B})$. There were no significant correlations between the presence of locoregional metastasis and the TRAb values.

\section{Outcome}

The outcome of the 154 Graves' disease patients with papillary thyroid cancer was compared with that of the 176 matched euthyroid patients with papillary thyroid cancer. The follow-up period ranged between 10.6 and 120.0 months (median 50.4) in the Graves' disease group and between 6.5 and 120.0 months (median 53.6) in the euthyroid group, and was very similar in the two groups $(P=0.4008)$. Table 2 shows the occurrence of distant and locoregional lymph node metastasis. During the follow-up period, one new case $(0.6 \%)$ of distant metastasis was observed in the Graves' disease group, and four new cases $(2.3 \%)$ were observed in the euthyroid group $(P=0.2284)$. At the end of the follow-up period, persistent distant metastasis was found in two Graves' disease patients and six euthyroid patients $(P=0.2137)$. Distant metastasis was the cause of death in one Graves' disease patient and two euthyroid patients $(P=0.925)$. The TRAb value of the Graves' patient who had a recurrence of thyroid cancer in the form of distant metastasis was $95.2 \%$.

Five Graves' disease patients $(3.2 \%)$ and fourteen euthyroid patients $(8.0 \%)$ experienced a relapse of cervical lymph node metastasis $(P=0.1518)$. Two of the Graves' disease patients had an immunologic remission before they developed lymph node metastasis. One new case $(0.6 \%)$ of cervical lymph node metastasis was observed in the Graves' disease group and four new cases $(2.3 \%)$ were observed in the euthyroid group $(P=0.1773)$. All of the patients with lymph node metastasis underwent metastatic lymph node dissection and remained disease-free until the final follow-up examination.

Seven Graves' disease patients with papillary thyroid cancer experienced a relapse of hyperthyroidism, but none of them developed thyroid cancer metastasis despite being positive for TRAb.

\section{Disease-free survival}

The disease-free survival rate of the patients who underwent thyroid surgery is shown in Fig 3. Microcarcinoma tends to have a good prognosis in Graves' disease patients. The differences in survival rate between the Graves' disease and euthyroid groups with microcarcinoma was not statistically significant (Fig 3A; $P=0.1118$ ). The difference in disease-free survival rate between the Graves' disease and euthyroid patients with thyroid cancer more than $1 \mathrm{~cm}$ also was not statistically significant (Fig. 3B; $P=0.7329$ ).

\section{Discussion}

Ultrasonography during the initial examination allowed identification of small cancers in the thyroid gland. It was possible that thyroid cancers were found at an earlier stage in Graves' disease patients. The patients who consulted our outpatients clinic with a complaint of a thyroid nodule during the same period as this study were older than the Graves' disease patients. Tumor size in the patients with a thyroid nodule was larger than in the Graves' disease patients (data not shown). The multifocality, lymph node metastasis, and distant metastasis status of the thyroid cancer patients, who also had Graves' disease, was similar to that of the euthyroid patients. Some investigators have reported 
finding multifocality and distant metastasis of thyroid cancer more frequently in Graves' disease patients than in euthyroid patients $(1,4)$. The discrepancies may be attributable to differences in the genetic background and/or iodine intake as an environmental factor (11).

High serum levels of thyroid-stimulating antibody have been reported to stimulate the growth of thyroid cancer metastasis (6). In this study, the TRAb values were not correlated with the multifocality or lymph node metastasis status of thyroid cancer. The TRAb values and tumor size were inversely correlated. The autoimmune process of Graves' disease may affect the clinical evolution of thyroid cancer by altering host immune responses. Immunologic remission can be achieved by surgery (12) and during antithyroid drug therapy (13). In this study, the patients who had an immunologic remission of Graves' disease developed cancer metastasis. Other Graves' disease patients who had continuously high serum TRAb levels did not develop cancer metastasis during the follow-up period, even though they relapsed into hyperthyroidism. Papillary thyroid cancer occurs regardless of the achievement of immunologic remission.

The difference between the disease-free survival rate during the follow-up period of the Graves' disease patients and euthyroid patients with papillary thyroid cancer was not significant. The presence of concomitant thyroid cancer is regarded as a factor for choosing a surgical treatment. We suggest that Graves' disease patients should undergo a detailed thyroid gland examination by ultrasonography. We do not have any indication that additional surgery or radioiodine therapy would have been useful in our patients with incidental papillary thyroid cancer. We concluded that the outcome of Graves' disease patients with papillary thyroid cancer is similar to that of euthyroid patients with papillary thyroid cancer.

\section{Acknowledgement}

We are grateful to K Okuwa and $\mathrm{H}$ Yoshimura for insight into the patient files; H Kamma for his advice; S Tamai, K Kameyama and K Yakumaru for diagnostic histopathology.

\section{References}

1 Belfiore A, Garofalo MB, Giuffrida D, Runello F, Filetti S, Fiumara A, Ippolito O \& Vigneri R. Increased aggressiveness of thyroid cancer in patients with Graves' disease. Journal of Clinical Endocrinology and Metabolism $1990 \mathbf{7 0} 830-835$.

2 Ozaki O, Ito K, Kobayashi K, Toshima K, Iwasaki H \& Yashiro T. Thyroid carcinoma in Graves' disease. World Journal of Surgery 199014 437-440.

3 Mazzaferri EL. Thyroid cancer and Graves' disease. Journal of Clinical Endocrinology and Metabolism 1990 70 826-829.

4 Pellegriti G, Belfiore A, Giuffrida D, Lupo L \& Vigneri R. Outcome of differentiated thyroid cancer in Graves' patients. Journal of Clinical Endocrinology and Metabolism 199883 2805-2809.

5 Roger P, Taton M, Van Sande J \& Dumont JE. Mitogenic effects of thyrotropin and adenosine $3^{\prime}, 5^{\prime}$-monophosphate in differentiated normal human thyroid cells in vitro. Journal of Clinical Endocrinology and Metabolism 198866 1158-1165.

6 Filetti S, Belfiore A, Amir SM, Daniels GH, Ippolito O, Vigneri R \& Ingbar SH. The role of thyroid-stimulating antibodies of Graves' disease in differentiated thyroid cancer. New England Journal of Medicine 1988318 753-759.

7 Katz SB, Garcia AJ \& Niepomniszcze H. Development of Graves' disease nine years after total thyroidectomy due to follicular carcinoma of the thyroid. Thyroid 19977 909-911.

8 Cantalamessa L, Baldini M, Orsatti A, Meroni L, Amodei V \& Castagnone D. Thyroid nodules in Graves disease and the risk of thyroid carcinoma. Archives of Internal Medicine 1999159 1705-1708.

9 Kikuchi S, Noguchi S, Yamashita H, Uchino S \& Kawamoto H. Prognosis of small thyroid cancer in patients with Graves' disease. British Journal of Surgery 200693 434-439.

10 Hales IB, McElduff A, Crummer P, Clifton-Bligh P, Delbridge L, Hoschl R, Poole A, Reeve TS, Wilmshurst E \& Wiseman J. Does Graves' disease or thyrotoxicosis affect the prognosis of thyroid cancer. Journal of Clinical Endocrinology and Metabolism $19927 \mathbf{5}$ 886-889.

11 Belfiore A, Russo D, Vigneri R \& Filetti S. Graves' disease, thyroid nodules and thyroid cancer. Clinical Endocrinology 200155 711-718.

12 Sugino K, Ito K, Ozaki O, Mimura T, Iwasaki H \& Wada N. Postoperative changes in thyrotropin-binding inhibitory immunoglobulin level in patients with Graves' disease: is subtotal thyroidectomy a suitable therapeutic option for patients of childbearing age with Graves' disease? World Journal of Surgery 199923 727-731.

13 Takasu N, Oshiro C, Akamine H, Komiya I, Nagata A, Sato Y, Yoshimura $\mathrm{H} \&$ Ito K. Thyroid-stimulating antibody and TSHbinding inhibitor immunoglobulin in 277 Graves' patients and in 686 normal subjects. Journal of Endocrinological Investigation 1997 $20452-461$.

Received 2 March 2007

Accepted 18 June 2007 